\title{
Un nouveau monde à bâtir pour le 21e siècle... ensemble
}

Écrit par : Anne-Lise Prigent, L'Observateur de l'OCDE

Dernière mise à jour : 13 juillet 2018

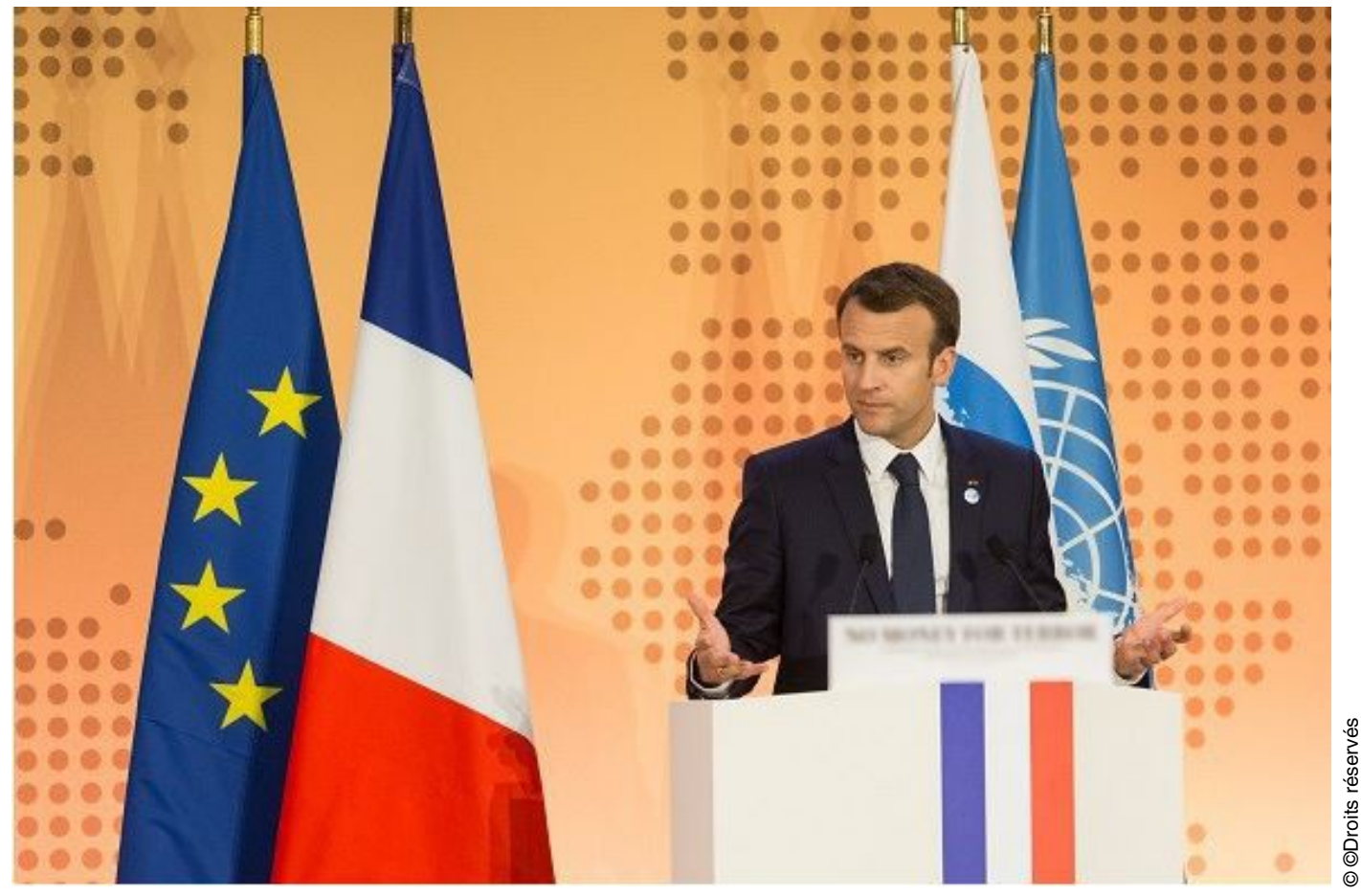

Notre monde semble être à la croisée des chemins, et avec lui, le multilatéralisme qui a été le socle de la coopération internationale depuis la 2 e Guerre mondiale. Où en sommesnous ? Comment aller de l'avant ? Les dernières discussions internationales apportent des éléments de réponse...

"J'ai un rendez-vous avec la mort. » Nous sommes en 1916. Ces paroles sont celles d'Alan Seeger, un jeune poète américain qui s'est engagé dans la légion étrangère. Il mourra pour la France et la liberté. Près de 20 millions de morts plus tard, cette première guerre mondiale se terminera enfin. Une autre la suivra une vingtaine d'années plus tard et fauchera entre 50 et 70 millions de vies.

Quelques décennies plus tard, nous voici en 2018. Emmanuel Macron s'adresse au Congrès américain. Ce n'est pas un hasard s'il commence son discours en parlant 
d'Alan Seeger. En évoquant les combats menés au nom de la liberté et de la démocratie, il parle aussi des risques qui nous guettent.

Pilier fondamental de la liberté et de la paix, le système multilatéral a été créé par les États-Unis, rappelle Emmanuel Macron devant le Congrès. Depuis la fin de la deuxième guerre mondiale, la coopération internationale a pu se développer grâce à ce multilatéralisme.

Or en 2018, alors que les défis économiques, sociaux et environnementaux se multiplient, la volonté de coopérer s'amenuise. Comme le rappelle Emmanuel Macron, nos valeurs sont attaquées. Le terrorisme rôde. La tentation du repli sur soi, de l'isolationnisme et du nationalisme gagne du terrain.

Et pourtant, 'the only thing to fear is fear itself.' (" La seule chose dont nous devons avoir peur est la peur elle-même. "). Ces mots sont de Roosevelt, cité par Emmanuel Macron. Autrement dit, la peur ne résout rien et n'empêche pas le danger, ni les mutations tectoniques de notre époque.

Ces défis mondiaux de notre temps-que nos ancêtres n'auraient sans doute même pas pu imaginer, comme le note Emmanuel Macron-nous n'avons d'autre choix que d'y répondre.

We need to redesign the way in which we co-operate in order to build a new world for the 21st century. In the spirit of multilateralism, we must listen to each other and move forward together. At a time when many feel betrayed by their elitesespecially since the 2008 crisis-many citizens no longer believe in these multilateral approaches. As Mr Gurría remarks, we need to reshape and strengthen multilateral institutions. We need to make globalisation work for everybody and for our planet. Our common action must deliver more tangible results to our societies.

Pour construire le nouvel ordre mondial du 21e siècle, il faudra réinventer notre manière de coopérer, de nous écouter les uns les autres et d'avancer ensemble dans l'esprit du multilatéralisme.

En matière de coopération, l'OCDE est un cas d'école. Angel Gurria, secrétaire général de l'organisation, se fait l'écho de l'inventeur de la pénicilline, Alexandre Fleming, qui disait que « plus le monde devient complexe, plus il est difficile d'accomplir quelque chose sans la coopération des autres ». Les défis complexes et planétaires auxquels nous faisons face nécessitent une vraie coopération.

Mais cette coopération doit évoluer et donner des résultats plus tangibles dans nos sociétés. À l'heure où beaucoup se sentent trahis par les élites, notamment depuis la crise, de nombreux citoyens ne croient plus en ces approches multilatérales. Comme le note M. Gurría, il faut renforcer, voire refonder, les institutions multilatérales, et rendre la mondialisation plus respectueuse de tous et de notre planète. 
Notre action commune doit avoir un impact réel, comme dans le cas de la lutte contre l'évasion fiscale avec ces 85 milliards d'euros récupérés pour les contribuables grâce à l'échange automatique d'informations. Comme le souligne M. Gurria, l'impact réel est aussi palpable à travers la Convention de l'OCDE contre la corruption, ou le Programme PISA, qui permet à 72 pays d'apprendre les uns des autres en matière d'éducation. Cet impact s'exprime encore à l'OCDE dans des domaines aussi divers que les principes directeurs de l'OCDE à l'intention des entreprises multinationales ou encore l'égalité entre hommes et femmes en matière d'éducation, d'emploi et d'entrepreneuriat.

Le multilatéralisme, c'est aussi une dynamique : celle de l'Accord de Paris sur le changement climatique ou celle des Objectifs du développement durable, autant de défis à relever pour les générations actuelles et futures. Et c'est bien sûr, en matière de justice fiscale, le projet BEPS de l'OCDE, qui constitue, comme le rappelle Emmanuel Macron, « une avancée sans précédent contre toutes les pratiques de transfert d'activité à des fins fiscales » et s'inscrit dans un cadre inclusif avec plus de 100 pays et territoires. «Il ne nous serait pas pardonné d'entraver la dynamique collective unique créée par le projet BEPS », prévient Emmanuel Macron, qui a présidé fin mai la réunion du Conseil de l'OCDE au niveau des Ministres autour du thème du multilatéralisme.

Le multilatéralisme, c'est notre socle commun, si naturel que nous oublions parfois qu'il nous soutient et nous relie, au risque de scier la branche sur laquelle nous sommes assis. C'est le meilleur moyen d'aller de l'avant, en continuant à nourrir le dialogue et la coopération dans un monde interdépendant. Ce formidable levier, ce catalyseur d'idées et de bonnes pratiques doit être mis à profit et, certes, réformé, mais non aboli.

Car enfin, le multilatéralisme n'est pas « un sport confortable, un jeu pour diplomates assis " : il faut, selon Emmanuel Macron, " sortir de son bureau, aller chercher d'autres énergies et représenter le monde tel qu'il est ».

Oui, la gouvernance multilatérale doit évoluer vers une dimension plus participative, mieux représenter les peuples et s'ancrer davantage aux échelons locaux et régionaux, prévient la professeure Laurence Boisson de Chazournes. Elle doit transcender les frontières et représenter des communautés de valeurs et d'intérêt.

Pour avancer, il est urgent de travailler sur nos convergences, et de profiter de la richesse de nos diversités pour bâtir notre monde commun.

Pour que les Alan Seeger de demain aient un rendez-vous avec la vie.

@L'Observateur de l'OCDE, juin 2018 


\section{Références}

Emmanuel Macron - Discours Inaugural de la Réunion du Conseil de l'OCDE au Niveau des Ministresh http://www.oecd.org/fr/forum/programme/\#!129311

Forum de l'OCDE 2018, 29-30 mai (sous l'égide de la France)http:// www.oecd.org/fr/forum/

Gurría A. (2018), "Multilateralism Is the Only Way Forward”, Project Syndicate

Gurría A. (2018), "La coopération internationale est le plus sûr moyen de construire des politiques meilleures pour une vie meilleure", L'Observateur de l'OCDE, Paris

French Ministry of Foreign Affairs and International Development, Le multilatéralisme peut-il encore préserver la paix? (Can multilateralism still preserve peace?)

Ansley R. (2017), “Making the Case for Mutilateralism”, Atlantic Council 